\title{
Estructura factorial y fiabilidad del Cyberbullying Questionnaire (CBQ) y su complemento (CBQ-V) en adolescentes ecuatorianos
}

\author{
Factorial Structure and Reliability of Cyberbullying Questionnaire \\ (CBQ) and her Complement (BCQ-V) in Ecuadorian Teenagers
}

\author{
Isabel Ramos-Noboa ${ }^{1}$ \\ Aitor Larzabal-Fernández ${ }^{2}$ \\ Rodrigo Moreta-Herrera ${ }^{3}$
}

1,2,3 Pontificia Universidad Católica del Ecuador, Escuela de Psicología,Ambato, Ecuador

Resumen. Objetivo. Analizar la estructura factorial y la fiabilidad del Cyberbullying Questionnaire (CBQ), que mide la perpetración del cyberbullying, y además medir su complemento, la victimización (CBQ-V) en una muestra de adolescentes tungurahuenses. Método. El estudio es de carácter instrumental para constatar la construcción factorial y la consistencia interna de las pruebas en una muestra de 998 estudiantes de bachillerato de seis centros educativos de Tungurahua. Resultados. Se encontró que el CBQ explica el 58.5\% de la varianza con tres factores con valores de $\mathrm{S}-\mathrm{B} \chi^{2}=29.5 ; g l=24 ; p>.05 ; \mathrm{S}-\mathrm{B} \chi^{2} / g l=1.2 ; \mathrm{NNFI}=.95 ; \mathrm{CFI}=.96 ; \mathrm{GFI}=.90 \mathrm{y} \mathrm{RMSEA}=.08 \mathrm{IC}$ $90 \%$ [.10 - .05]. Mientras que el CBQ-V igualmente con tres factores explica el 52.6\% de la varianza con S-B $\chi^{2}=$ $29.5 ; g l=24 ; p>.05 ; \mathrm{S}-\mathrm{B} \chi^{2} / g l=1.2 ; \mathrm{NNFI}=.95 ; \mathrm{CFI}=.96 ; \mathrm{GFI}=.90$ y RMSEA $=.08 \mathrm{IC} 90 \%$ [.10 - .05]. En cuanto a la fiabilidad, los puntajes alcanzados para el CBQ es $\alpha_{\text {(ordinal) }}=.903$ y CBQ-V es $\alpha_{\text {(ordinal) }}=.879$. Se concluye que las pruebas bajo análisis son válidas como constructo y fiables en estudiantes de bachillerato de Tungurahua.

Palabras clave. Análisis factorial, cyberbullying, fiabilidad, perpetración, victimización.

Abstract. Objective. To analyze the factor structure and reliability of the Cyberbullying Questionnaire (CBQ), which measures the prevalence of cyberbullying; as well as measure the complement on victimization (CBQ-V). Method. The study is instrumental in verifying the factorial construction and internal consistency of the tests in a sample of 998 high school students from six educational centres in Tungurahua. Results. It was found that the CBQ explains $58.5 \%$ of the variance with three factors with values of $\mathrm{S}-\mathrm{B} \chi^{2}=29.5 ; d f=24 ; p>.05 ; \mathrm{S}-\mathrm{B} \chi^{2} / d f=1.2$; $\mathrm{NNFI}=.95 ; \mathrm{CFI}=.96 ; \mathrm{GFI}=.90$ and RMSEA $=.08 \mathrm{IC} 90 \%$ [.10 - .05]. While the CBQ-V also with three factors explains $52.6 \%$ of the variance with S-B $\chi^{2}=29.5 ; d f=24 ; p>.05 ; \mathrm{S}-\mathrm{B} \chi^{2} / d f=1.2$; NNFI $=.95$; CFI $=.96$; GFI $=.90$ and RMSEA $=.08$ IC 90\% [.10 -.05]. Regarding reliability, the scores reached for the CBQ is $\alpha_{\text {(ordinal) }}=.903$ and CBQ-V is $\alpha_{\text {(ordinal) }}=.879$. It is concluded that the tests under analysis are valid as a construct and reliable in Tungurahua high school students.

Keywords. Factor analysis, cyberbullying, reliability, perpetration, victimization.

${ }^{1}$ Isabel Ramos-Noboa. Pontificia Universidad Católica del Ecuador, Escuela de Psicología. Dirección Postal: Av. Manuela Sáenz s/n, Sector el Tropezón, Sede Ambato, Ecuador. E-mail: iramos@pucesa.edu.ec

${ }^{2}$ Aitor Larzabal-Fernández. Pontificia Universidad Católica del Ecuador, Escuela de Psicología, Ambato, Ecuador. E-mail: alarzabal@pucesa.edu.ec ${ }^{3}$ Rodrigo Moreta-Herrera. Pontificia Universidad Católica del Ecuador, Escuela de Psicología, Ambato, Ecuador. E-mail: rmoreta@pucesa.edu.ec

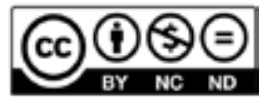

Esta obra está bajo una licencia de Creative Commons Reconocimiento-NoComercial-SinObraDerivada 4.0 Internacional. 


\section{Introducción}

La violencia escolar es un fenómeno arraigado en los sistemas educativos de diversos países y cuenta con distintas formas de manifestación (di Napoli, 2016). Una de estas formas es el bullying o 'acoso escolar', el cual comprende una forma específica de maltrato o violencia psicológica, verbal o física, entre escolares en los centros de enseñanza. En el que se molesta, hostiga y atormenta sistemáticamente a un individuo durante distintos períodos de tiempo (Olweus, 1998) y con múltiples efectos negativos en las víctimas (Garaigordobil, 2011).

Entre las distintas modalidades de ejecución y debido al auge y al acceso que tienen las tecnologías virtuales, existe el acoso escolar que se extiende al mundo cibernético. El Cyberbullying (CB) o 'acoso escolar cibernético', comprende además, el uso de las TIC's (Internet, computador, Tablet, móvil, otros) para ejercer acoso, persecución y hostigamiento entre iguales a través de la difusión de contenido específico de la víctima (Garaigordobil, 2015) con el fin de agredirla, humillarla o generar un efecto lesivo. Este fenómeno es uno de los factores que a la larga, puede tener notoria en la elevación del riesgo en la salud física y mental en escolares (Moreta, Reyes, Mayorga, \& León-Tamayo, 2017).

El CB podría considerarse una extensión del bullying tradicional aplicado al aspecto virtual. Sin embargo, existen diferencias que la tratan como una manifestación distinta de violencia escolar. Por ejemplo, en el anonimato del acosador (Beckman, Hagquist, \& Hellström, 2012), la extensión del acoso más allá del entorno escolar (Wachs, 2012), en la hora del día (Tokunaga, 2010), y en el potencial del mensaje agresivo por medio de palabras, imágenes, vídeos y otros; así como en la difusión del acoso a varias personas (García \& Orellana, 2010).

Existen diversos tipos o formas de generar CB, siendo la única limitación la imaginación del agresor y la pericia tecnológica para el uso del acoso (Garaigordobil, 2011). Así tenemos: a) peleas online o Flaming; b) envío de rumores contra la reputación de alguien; c) robos de cuentas de redes sociales para envío de mensajes que dañen a la víctima; d) difusión de secretos por redes sociales; e) exclusión deliberada de grupos online; f) envío repetido de mensajes amenazantes (Calvete et al., 2010); g) cargas en internet con imágenes comprometedoras (reales o montajes); h) inscripción de las víctima en webs clasificadoras de personas por sus aspectos negativos a través de votos; i) dar de alta la dirección de e-mail de la víctima en webs para recepción de spam (Flores, 2008), entre otros.

Las consecuencias negativas en víctimas expuestas al CB se relacionan con problemas en torno al rendimiento académico (Ortega-Reyes \& González-Bañales, 2015); y psicológicos, tales como: ansiedad, depresión, estrés, somatizaciones, suicidios, violencia (Garaigordobil, 2011; Fisher, Gardella, \& Teurbe, 2016), soledad (Eden, Heiman, \& Olenik, 2016), estrés percibido (Larzabal-Fernandez, Ramos-Noboa, \& Hong, 2019) y poca satisfacción con la vida (You, Lee, \& Kim, 2016). Mientras que, desde el lado del victimario se encuentra relación con la reputación social y la generación de conductas antisociales (Buelga, Iranzo, Cava, \& Torralba, 2015).

En países europeos como España, las prevalencias del CB bordean el 30\% en conductas perpetradoras (Estévez et al., 2010; Garaigordobil \& Aliri, 2013; García \& Jiménez, 2010; Buelga et al., 2015).Ya en los países de la región sudamericana, como Chile (Varela et al., 2014) y Perú (García \& Orellana, 2010), las prevalencias de conductas perpetradoras 
fluctúan entre el 11\% y 12\% respectivamente. Y en Ecuador, de manera moderada bordea el $2.5 \%$ para la perpetración y el $2.3 \%$ en victimización moderada (Moreta-Herrera, Poveda-Ríos, \& Ramos-Noboa, 2018).

Evaluación de Cyberbullying

Actualmente existen diversos instrumentos que nos permiten evaluar a modo de autoreporte agresores o víctimas del CB. Entre ellos por ejemplo, el European Cyberbullying Intervention Project Questionnaire (ECIPQ) (Del Rey et al., 2015); la Escala de Cyberbullying (Cyb-agress) que evalúa conductas de agresiones (Buelga \& Pons, 2012); el Cuestionario Cyberbullying (Ortega, Calamaestra, \& Mora, 2008); El Test Cyberbullying (Garaigordobil, 2013; 2015); el Cyberbyllying scale (Erdur-Baker, 2010), entre otros. No obstante, el principal problema consiste en que estos se han desarrollado en el contexto europeo y anglosajón; y las experiencias evaluativas en el contexto latinoamericano y en español son limitadas. Por lo que existe una dificultad en la generalización del constructo a medir y el contraste de los resultados con otras realidades y culturas.

En este aspecto, si destaca el Cyberbullying Questionnaire (CBQ, Calvete et al., 2010), centrado en el agresor y el complemento en la victimización tras la perpetración (CBQ-V, Estévez et al., 2010). Que, aunque diseñados en España, cuenta con resultados positivos de adaptación en países como México (Castellanos, George, \& Gámez, 2017), Perú (Peña, Urday, \& Soto, 2013), Argentina (Resett, 2016) y Ecuador (Moreta-Herrera, Poveda-Ríos, $\&$ Ramos-Noboa, 2018). Por lo que esta herramienta tiene una potencial eficiencia para el campo de evaluación del CB.

El CBQ se presenta como un cuestionario de 16 ítems con una estructura unifactorial, y una explicación de la varianza del 44.1\%. Mientras que el CBQ-V está conformado por 11 ítems con estructura factorial unidimensional. Estos se evidencian como instrumentos de adecuada estructuración factorial y de elevada fiabilidad (medidas con el Coeficiente Alfa de Cronbach) en muestras españolas. En el caso de México (GámezGuadix, Villa-George, \& Calvete, 2014), se observa que las pruebas funcionan como una solución bifactorial correlacionada (aunque el CBQ y el CBQ-V fueron diseñadas para valoraciones independientes). Si bien es cierto que los valores de ajuste son pertinentes, la fiabilidad arrojó resultados ambiguos con valores de $\alpha=.81$ para el CBQ y $\alpha=.59$ para el CBQ- $V$. Finalmente, en Argentina se encontró que la escala funciona de manera integral conformada por dos factores del CBQ y uno del CBQ-V, con valores de fiabilidad elevados (Resett \& Gámez-Guadix, 2018).

Aunque existe evidencia de validez de la prueba, existen tres problemas de orden metodológico a identificar. El primero tiene que ver con el carácter unifactorial del CBQ y el CBQ-V extendido por Latinoamérica; en el cual se da por hecho la estructura factorial de estos; aun cuando la evidencia refleja que no es así (Resett \& Gámez-Guadix, 2018). Y evita la necesidad de verificar la estruturación factorial. El segundo problema guarda relación con la fiabilidad de la prueba, pues como sucede habitualmente en la verificación de este, se hace un uso inadecuado del estadístico de Cronbach $(\alpha)$. Pues, la naturaleza del estadístico es para su uso en variables continuas, pero en las pruebas mencionadas los ítems se conciben como variables categóricas. Y el tercero, es que la mayoría de 
investigaciones recogen estudios únicamente de validez de constructo de la prueba y de fiabilidad, dejando a un lado otros procesos de validación como los de convergencia, divergencia, discriminante y de temporalidad. En conjunto, el no tratamiento de estos problemas instrumentales puede traer problemas en el proceso de generalización de resultados en el primer caso, así como estimaciones sobre fiabilidad erróneas e inexactas en el segundo caso (Domínguez-Lara, 2014; Dominguez-Lara, 2017).

Por lo tanto, con estos antecedentes es necesaria la realización de estudios confirmatorios con mayor rigurosidad científica y técnica, así como adaptados a la realidad del Ecuador. En el presente estudio se abordará los estudios de validez factorial y fiabilidad, dejando a futuras investigaciones el análisis de otras propiedades psicométricas.

A la luz de la evidencia se plantean como objetivos del estudio: a) Conocer sobre la estructuración factorial que tienen los cuestionarios CBQ y CBQ-V en adolescentes ecuatorianos; y b) Determinar el nivel de fiabilidad que guardan las pruebas en mención. De estos objetivos se hipotetiza por una parte que la estructura factorial varía del modelo original, no conformando una estructura de dos factores correlacionados y con varios factores independientes entre los distintos test; y que las consistencias internas de las pruebas son adecuadas.

\section{Método}

\section{Diseño}

El presente estudio se enmarca en el criterio empírico cuantitativo (Ato, López, \& Benavente, 2013), como un estudio instrumental que tiene como fin conocer la estructura factorial y la fiabilidad de las puntuaciones entre los ítems componentes de las pruebas de perpetración del cyberbullying (CBQ) y de victimización (CBQ-V).

\section{Participantes}

La población de referencia del estudio comprende a los estudiantes de bachillerato de la provincia de Tungurahua, Ecuador; y que asisten al sistema educativo público y privado. Tomando como referencia el año de aplicación se trataron aproximadamente 39.808 sujetos (Instituto Nacional de Estadísticas y Censos [INEC], 2015). Para el presente estudio se establece un nivel de confianza del $99 \%$ y un margen de error del $5 \%$, por lo que la muestra al menos debe estar conformada por 655 casos.

La participación total de la muestra estuvo conformada por 998 casos. Estudiantes de los distritos educativos 1 y 2 de la ciudad de Ambato, Ecuador. Con edades entre los 14 a los 19 años $(M=15.47$ años; $D T=1.17)$. Un $47.2 \%$ de hombres y un $51.7 \%$ de mujeres. Los participantes provienen de seis unidades educativas $(70.8 \%$ de unidades públicas y $29.2 \%$ de privadas). Cursan el primer (44.3\%), segundo (30\%) y tercer (25.6\%) año de bachillerato. La selección de los participantes se realizó a través de un muestreo probabilístico, aleatorio estratificado, considerando la variable de tipo de unidad educativa (pública y privada).

\section{Instrumentos}

Cyberbullying Questionnaire (CBQ, elaborado por Calvete et al., 2010). Cuenta con 16 ítems correspondientes que evalúan distintas formas de acoso cibernético. Es decir, la 
perpetración a la que son sometidas las víctimas. En el presente estudio no se tuvieron en cuenta dos de los ítems, pues estos están relacionados a una respuesta escrita de otros tipos de acoso (Gámez-Guadix, Villa-George, \& Calvete, 2014).

Cuestionario de víctimas de Cyberbullying (CBQ-V, complemento del CBQ, elaborado por Estévez et al., 2010). Para medir la victimización con respecto al cyberbullying. O sea, la forma y la afectación que tiene tras la violentación por el o los perpetradores. De la misma manera que en el CBQ, se retiraron dos ítems de respuesta escrita sobre el tipo de acoso (Gámez-Guadix, Villa-George, \& Calvete, 2014).

\section{Procedimiento}

El Distrito de Educación 1 y 2 del cantón Ambato autorizó el trabajo para las unidades educativas públicas y en las instituciones privadas se coordinó el trabajo directamente con las autoridades. La herramienta fue adaptada lingüísticamente cambiando términos que se usan habitualmente en el español de España, como "móvil" por el español de Ecuador "celular". El proceso se realizó contando con la presencia de psicólogos de procedencia española y ecuatoriana para analizar la equivalencia de términos. Una vez creada la versión de la herramienta, se aplicó a 30 estudiantes de primero de bachillerato, con el fin de analizar la comprensión de la misma a manera de prueba piloto. Para su aplicación se creó una página web (www.cuestionariospucesa.com) enlazada a la herramienta generadora de cuestionarios de Google drive. En la página web se añadieron ítems sociodemográficos (edad, sexo, unidad educativa y año de bachillerato) y el consentimiento informado. Se procedió a visitar las diferentes unidades educativas entre septiembre del 2015 y marzo del 2016 para aplicar las herramientas; con lo que se consiguió un total de 1038 encuestas. Posteriormente se realizó un análisis y depuración de respuestas incompletas o consideradas falsas (ya sea por edades imposibles o unidades educativas inexistentes) con un total de 998.

Seguidamente, se procedió a la sistematización de la información en bases de datos electrónicas para la gestión estadística y la obtención de resultados; las cuales se sintetizaron en informes de investigación y en el presente trabajo de divulgación científica. Previa a su publicación se obtuvo el consentimiento de la comisión de publicaciones de la Pontificia Universidad Católica del Ecuador Sede Ambato, la cual ejerce funciones de revisión ética de los procesos investigativos.

\section{Análisis de datos}

El tratamiento estadístico se basa en tres grupos de análisis. El primero sobre los valores medios, desviación y distribución alcanzados en los ítems de las escalas de perpetración y victimización. Con ello se busca comprobar el supuesto de normalidad univariante, el mismo que se confirma si los puntajes de la asimetría y la curtosis se encuentran dentro del rango de normalidad $( \pm 1.5)$ (Ventura \& Caycho, 2016). También se verifica la validez de constructo con el Coeficiente de Correlación ítem-total corregido $\left(r_{(i-1) t}\right)$ y se estima si los ítems son pertinentes para cada caso.

El segundo análisis comprende la revisión de la estructuración factorial a través del Análisis Factorial Exploratorio (AFE) con 749 participantes (75\% de la muestra); y el Análisis Factorial Confirmatorio (AFC) con 249 participantes (25\% restante), que comprenden 
tamaños adecuados para estos análisis (Brown, 2015). Con esto se garantiza la "no representatividad" de la muestra (Ferrando \& Anguiano-Carrasco, 2010) y confirmar el modelo a través de la independencia de resultados. El AFE con correlaciones policóricas (Domínguez, 2014) incluye los análisis preliminares de adecuación muestral con la prueba Kayser-Meyer-Olkin (KMO) y la conformación de factores con la prueba Bartlett $\left(\chi^{2}\right)$. $\mathrm{El}$ análisis de comunalidades $\left(h^{2}\right)$ para verificar homocedasticidad o baja comunalidad. Y también la extracción de factores por el Método de Mínimos Cuadrados no Ponderados (MCNP) con la rotación oblicúa (Promin), debido a la ausencia de normalidad multivariada.

El AFC con el Método de Máxima Verosimilitud (MMV) por otra parte, incluyó la evaluación de la estimación robusta con la prueba de Satorra-Bender $\left(\mathrm{S}-\mathrm{B} \chi^{2}\right)$ en casos de no normalidad multivariante (Satorra \& Bentler, 2001) y la razón Chi cuadrado normada (S-B $\chi^{2} / g l$ ) (Hu \& Bentler, 1999). Mientras que las pruebas de bondad de ajuste como el Índice de Ajuste no normalizado (NNFI), el Índice de Ajuste Comparativo (CFI) y el Índice de Bondad de Ajuste (GFI) son elevadas cuando las puntuaciones son iguales o superiores a 9 (Byrne, 2006; Batista-Fogueta, Coenders, \& Alonso, 2004), así como cuando el Error cuadrático medio de Aproximación (RMSEA) es menor a .08 (Abad, Olea, Ponsoda, \& García, 2011).

Finalmente se incluye un análisis de la fiabilidad entre las puntuaciones obtenidas en los ítems que componen las distintas escalas analizadas. Para ello se utilizó tres cálculos de fiabilidad: a) El Coeficiente Alfa de Cronbach, b) El Coeficiente Alfa ordinal ( $\left.\alpha_{\text {(ordinal }}\right)$ y c) El coeficiente Omega de McDonald ( $\omega$, McDonald, 1999) el cual se utiliza como método confirmatorio del $\alpha_{\text {(ordinal) }}($ Elosua \& Zumbo, 2008). A más de ello se hace un cálculo de la atenuación (Dominguez-Lara, 2017) existente entre los cálculos de $\alpha$ y $\alpha_{\text {(ordinal) }}$ para conocer el porcentaje de minusvaloración de la fiabilidad entre la prueba de Cronbach con respecto al alfa ordinal. La gestión informática de los datos se realizó con la utilización del software R Commander 2.2-3 (R Core Team, 2015) para los análisis descriptivos, comparativos y el AFC y el programa FACTOR 9.2 (Lorenzo-Seva \& Ferrando, 2013) para el cálculo de las correlaciones policóricas para la fiabilidad de las escalas. Los programas informáticos son de libre acceso al público.

\section{Resultados}

\section{Análisis preliminar}

El análisis preliminar, como se observa en la Tabla 1, muestra en el CBQ y el CBQ-V que los puntajes medios obtenidos son homogéneos con tendencia a los valores bajos. Mientras que a nivel de evaluación de asimetría y curtosis muchos de los ítems sobrepasan el criterio $( \pm 1.5)$, por lo que la confirmación del supuesto de normalidad univariada se rechaza. Con respecto a la validez de constructo que tienen los ítems para los cuestionarios, estos se encuentran dentro de los rangos adecuados. Por lo que a partir de estos resultados es innecesaria la eliminación de ítems.

Análisis de estructuración factorial

En la evaluación del CBQ que trata de la perpetración, los análisis muestran que los comportamientos de los ítems presentan un ajuste adecuado, tanto para la exploración 
Tabla 1

Análisis de normalidad multivariada del CBQ y el CBQ-V $(n=998)$

\begin{tabular}{cccccc}
\hline Ítems & Media & Varianza & Asimetría & Curtosis & $r_{(i-t)}$ \\
\hline Perpetración & & & & & \\
Ítem 1 & .15 & .37 & 2.09 & 2.99 & .34 \\
Ítem 2 & .15 & .37 & 2.31 & 4.42 & .43 \\
Ítem 3 & .13 & .35 & 2.79 & 7.39 & .30 \\
Ítem 4 & .09 & .30 & 3.23 & 9.85 & .36 \\
Ítem 5 & .15 & .38 & 2.51 & 5.71 & .44 \\
Ítem 6 & .11 & .33 & 3.00 & 8.61 & .28 \\
Ítem 7 & .10 & .31 & 3.01 & 8.27 & .41 \\
Ítem 8 & .14 & .36 & 2.44 & 5.12 & .33 \\
Ítem 9 & .38 & .55 & 1.05 & .07 & .40 \\
Ítem 10 & .08 & .29 & 3.67 & 13.66 & .44 \\
Ítem 11 & .44 & .57 & .92 & -.15 & .46 \\
Ítem 12 & .07 & .27 & 3.76 & 14.09 & .29 \\
Ítem 13 & .28 & .48 & 1.40 & 0.87 & .35 \\
Ítem 14 & .04 & .22 & 6.03 & 39.49 & .25 \\
Victimización & & & & & \\
Ítem 1 & .12 & .36 & 3.00 & 8.81 & .34 \\
Ítem 2 & .13 & .36 & 2.74 & 7.06 & .37 \\
Ítem 3 & .04 & .21 & 5.82 & 36.79 & .35 \\
Ítem 4 & .10 & .32 & 3.13 & 9.57 & .26 \\
Ítem 5 & .23 & .46 & 1.79 & 2.34 & .29 \\
Ítem 6 & .05 & .25 & 4.90 & 25.74 & .42 \\
Ítem 7 & .05 & .24 & 5.38 & 31.22 & .40 \\
Ítem 8 & .08 & .28 & 3.46 & 11.59 & .38 \\
Ítem 9 & .15 & .41 & 2.78 & 7.37 & .33 \\
\hline Nota.r & & & &
\end{tabular}

Nota. $r_{(-1-t)}=$ Correlación ítem-test corregida.

factorial $\mathrm{KMO}=.81$; como para la constitución de factores $\chi^{2}=1689.6 ; p<.001$. Además, el análisis de comunalidades $\left(b^{2}\right)$ guarda correspondencia adecuada que permite descartar homocedasticidad entre los ítems. Ya en el AFE, como se observa en la Tabla 2, la conformación del CBQ con tres factores $(40.1 \%, 10.2 \%$ y $8.2 \%)$, explica en conjunto una varianza del 58.5\%. Además, todas las cargas factoriales de los ítems agrupados se encuentran por encima o igual a .3 .

Por otra parte, en lo que respecta al CBQ-V, encargado de realizar una valoración de victimización sobre la perpetración virtual, los indicadores preliminares $\mathrm{KMO}=.70 \mathrm{y} \chi^{2}$ $=826 ; p<.001$ para la adecuación y conformación de factores, por lo que son adecuados 
Tabla 2

Análisis factorial Exploratorio del CBQ

\begin{tabular}{|c|c|c|c|c|}
\hline Ítems & $b^{2}$ & 1 & 2 & 3 \\
\hline Ítem 01 & .552 & .974 & & \\
\hline Ítem 02 & .598 & .840 & & \\
\hline Ítem 10 & .658 & .473 & & \\
\hline Ítem 03 & .277 & .313 & & \\
\hline Ítem 05 & .743 & & .984 & \\
\hline Ítem 04 & .519 & & .743 & \\
\hline Ítem 07 & .546 & & .709 & \\
\hline Ítem 14 & .334 & & .565 & \\
\hline Ítem 06 & .386 & & .538 & \\
\hline Ítem 08 & .279 & & .300 & \\
\hline Ítem 13 & .611 & & & .926 \\
\hline Ítem 11 & .570 & & & .636 \\
\hline Ítem 12 & .397 & & & .601 \\
\hline Ítem 09 & .315 & & & .327 \\
\hline$\%$ de varianza explicada & & .401 & .102 & .082 \\
\hline
\end{tabular}

Nota KMO $=.81 ;$ Bartlett $=1970.6 ; p<.001 ;$ Extracción: Mínimos cuadrados no ponderados; Rotación: Oblimin.

para los procesos futuros. También el análisis de comunalidades entre los ítems $\left(b^{2}\right)$, señala que se encuentran dentro de los parámetros esperados.

Los resultados del AFE, como se observa en la Tabla 3, muestran una conformación de tres factores $(19.8 \%, 16.9 \%$ y $15.7 \%)$, que de manera global permiten una explicación de la varianza del $52.6 \%$. Al igual que en el análisis anterior, estos resultados son adecuados.

\section{Análisis factorial confirmatorio}

En el presente análisis, como se observa en la Tabla 4, se busca confirmar los mejores modelos de ajuste factorial del CBQ y el CBQ-V. Para ello se analiza tanto los modelos referenciales como los empíricos obtenidos en los AFE.

En el AFC sobre el CBQ (Calvete et al., 2010), se encontró el mejor ajuste en el modelo de tres factores en el que se encontró ajustes robustos corregidos adecuados, así como en los distintos índices de ajuste (ver Tabla 4), por lo que se concluye que el modelo factorial es pertinente. Sobre el CBQ-V (Estévez et al., 2010), el ajuste robusto corregido del modelo es considerado como óptimo, así como también los índices complementarios.

Sobre la estructuración combinada propuesta por Gámez-Guadix, Villa-George, y Calvete (2014), no se puedo evidenciar que dicho ajuste sea pertinente ni en un modelo de CBQ y CBQ-V como dos factores correlacionados, ni a través de dos factores (Perpetración y Victimización) como factores de segundo orden. 
Tabla 3

Análisis factorial Exploratorio del CBQ-V

\begin{tabular}{lcccc}
\hline \multicolumn{1}{c}{ Ítems } & $h^{2}$ & 1 & 2 & 3 \\
\hline Ítem 04 & .452 & .666 & & \\
Ítem 01 & .460 & .658 & & \\
Ítem 02 & .476 & .650 & & \\
Ítem 03 & .516 & .643 & & \\
Ítem 06 & .741 & & .833 & \\
Ítem 07 & .699 & & .815 & \\
Ítem 05 & .587 & & & .765 \\
Ítem 08 & .444 & & & .620 \\
Ítem 09 & .358 & .198 & .169 & .565 \\
\% de varianza explicada & & & & .157 \\
\hline
\end{tabular}

Nota KMO $=.72 ;$ Bartlett $=1164.0 ; p<.001 ;$ Extracción: Mínimos cuadrados no ponderados; Rotación: Oblimin.

Tabla 4

Análisis Factorial Confirmatorio de las Escalas $C B Q, C B Q-V$

\begin{tabular}{|c|c|c|c|c|c|c|c|}
\hline Modelo & $S-B \chi^{2}$ & $g l$ & $\mathrm{~S}-\mathrm{B} \chi^{2} / \mathrm{gl}$ & NNFI & CFI & GFI & RMSEA \\
\hline \multirow[t]{2}{*}{ CBQ (Calvete et al., 2010) } & $128.1^{* * *}$ & 77 & 1.7 & .70 & .74 & .89 & .08 \\
\hline & & & & & & & {$[.10-.07]$} \\
\hline \multirow[t]{2}{*}{ CBQ (tres factores) } & $88.4^{* * *}$ & 74 & 1.2 & .91 & .93 & .92 & .06 \\
\hline & & & & & & & {$[.08-.05$} \\
\hline \multirow{2}{*}{$\begin{array}{l}\text { CBQ-V (Estévez et al., } \\
\text { 2010) }\end{array}$} & $52.8^{* * *}$ & 27 & 2.0 & .75 & .81 & .90 & .12 \\
\hline & & & & & & & {$[.14-.10]$} \\
\hline \multirow[t]{2}{*}{ CBQ-V (tres factores) } & $29.5^{* * *}$ & 24 & 1.2 & .95 & .96 & .90 & .08 \\
\hline & & & & & & & {$[.10-.05]$} \\
\hline \multirow{2}{*}{$\begin{array}{l}\text { CBQ + V (Gámez et al., } \\
\text { 2014) }\end{array}$} & $432.4^{* *}$ & 229 & 1.9 & .60 & .63 & .77 & .10 \\
\hline & & & & & & & {$[.12-.09]$} \\
\hline \multirow{2}{*}{$\begin{array}{l}\text { Dos factores de segundo } \\
\text { orden }\end{array}$} & $665.6^{* * *}$ & 223 & 3.0 & .54 & .63 & .81 & .09 \\
\hline & & & & & & & {$[.10-.08]$} \\
\hline
\end{tabular}

Nota. Método: Máxima Verosimilitud.

${ }^{* *} \mathrm{p}<0.01{ }^{* * *} \mathrm{p}<0.001$ 
Tabla 5

Análisis de fiabilidad del CBQ, el CBQ-V y el modelo integrado $C B Q$

\begin{tabular}{lccccc}
\hline \multicolumn{1}{c}{ Escalas } & Ítems & $\alpha$ & $\alpha_{(\text {ordinal) }}$ & $\omega$ & Atenuación \\
\hline CBQ (tres factores) & 14 & .797 & .903 & .904 & $12 \%$ \\
CBQ-V (tres factores) & 9 & .722 & .877 & .879 & $8 \%$ \\
\hline
\end{tabular}

Nota. $\alpha$ : Alfa de Cronbach; $\alpha_{\text {(ordinal) }}$ : Alfa de Zumbo; $\omega$ : Omega de Mcdonald.

Es decir, que el modelo en la muestra de adolescentes ecuatorianos tiene un mejor ajuste a través de la conformación de tres factores para cada uno de los instrumentos analizados.

Análisis de fiabilidad de los items y atenuación

Se encontró que la fiabilidad en el CBQ es elevada en los tres análisis de fiabilidad, el alfa ordinal y el coeficiente omega muestra valores de $\alpha_{\text {(ordinal) }}=.903 \mathrm{y} \omega=.904$; la atenuación es del 12\% de infravalorización de la fiabilidad del coeficiente de Cronbach. En el CBQ-V la fiabilidad resultó igualmente ser elevada con valores de $\alpha_{\text {(ordinal) }}=.877 \mathrm{y} \omega=.879$ con una atenuación del 8\%. De esta manera se estima que la fiabilidad es pertinente (ver Tabla 5).

\section{Discusión}

El estudio tuvo como objetivos el determinar la estructura factorial de las pruebas CBQ y CBQ-V para procesos de evaluación de cyberbullying en adolescentes escolarizados del Ecuador; tanto como perpetradores como víctimas adolescentes. A más de identificar la fiabilidad de las pruebas con métodos alternativos ajustados a la respuesta al ítem de tres opciones.

Inicialmente, se muestra una presencia leve aunque constante de conductas de perpetración y de CB entre los participantes del estudio, en la cual los ítems 9 (grabación de vídeos), 11 (envío de mensajes amenazantes) y 13 (envío de imágenes) son los de mayor magnitud. Así mismo, se detectó niveles bajos de victimización ante la perpetración de $\mathrm{CB}$, en el que resaltaron con mayor prevalencia los ítems 5 (recepción de mensajes amenazantes) y 9 (recepción de mensajes muy amenazantes). La baja presencia de este tipo de comportamientos (perpetración y victimización) concuerda con estudios preliminares hechos en adolescentes de Perú (García \& Orellana, 2010), Chile (Varela et al., 2014), Argentina (Resett, 2016) y Ecuador (Moreta-Herrera, Poveda-Ríos, \& Ramos-Noboa, 2018).

No se encontró distribución normal en las respuestas a los ítems, por lo que la normalidad univariante no fue cumplida. Así, el uso de análisis estadísticos más pertinentes son los no centrados en la media. Este aspecto de distribución se explica por la naturaleza del CB, al encontrarse baja variabilidad, señala grupos pequeños, pero específicos de agresores y víctimas identificadas. Mientras que la restante proporción es una mezcla poco definida de conductas manifiestas (e.g. agresores que posteriormente son víctimas y viceversa). Sobre la validez de constructo se evidencia que aportan significativamente cada uno de los ítems y son no redundantes.

Respecto a la estructuración factorial, en el CBQ tras el AFE con el método MCNP, la prueba se constituye con tres factores que explican el 58.1\% de la varianza. El AFC, 
además, confirma el ajuste del modelo. Estos resultados difieren de los hallazgos iniciales de Calvete et al., (2010); pues se encontró que la explicación de la varianza con tres factores es muy superior al ajuste inicial del $44.1 \%$ de manera unifactorial. De la misma manera, en el caso del CBQ-V el mejor ajuste se lo encuentra con una configuración de tres factores que explican el 52.6\% la varianza. También estos resultados difieren con los propuestos originalmente por Estévez et al., (2010), a través de un modelo unifactorial.

No se tiene claro las diferencias en estudios previos sobre la adecuación factorial de las pruebas y se requerirán más estudios complementarios para determinar aquello. Se estima que las variantes culturales y de acceso tecnológico por parte de los adolescentes son los que pueden influir en el fenómeno del cyberbullying; y a la vez en la construcción del mismo. Lo que se pudo determinar es la dinámica de funcionamiento de los factores. Así, en el CBQ el factor 1 se relaciona con el envío de mensajes y graficos a terceros; el factor 2 con la violación de la privacidad de la víctima para la humillación, y el factor 3 con el envío de grabaciones en situaciones comprometedoras a terceros. Mientras que en el CBQ-V el factor 1 tiene que ver con ser grabado, así como el envío de vídeos en situaciones humillantes, agresiones o en cicunstancias de contenido sexual; el factor 2 con la colocación de imágenes o comentarios humillanes en internet; y el factor 3 con el envío de mensajes amenazantes y el uso de la identifidad de la víctima. Se requieren mayores estudios que amplien la perspectiva de este fenómeno.

No se pudo evidenciar que la combinación del CBQ y el CBQ-V como un modelo factorial combinado propuestos por Gámez-Gaudix et al., (2014) en poblacion mexicana pueda replicarse en participantes ecuatorianos. Así, los estudios originales en españoles (Calvete et al., 2010; Estévez et al., 2010), muestras peruanas (Peña, Urday, \& Soto, 2013) y argentinas (Resett, 2016), junto con los hallazgos presentes, consideran oportuna la exclusiva evaluación de dichas condiciones de manera independiente.

Cabe señalar por otra parte, el tratamiento dado a los análisis factoriales y que no se consideró en los estudios (Calvete et al., 2010; Estévez et al., 2010; Gámez et al., 2014) al tratar modelos confirmatorios. Pues a diferencia de este estudio no se dividió a los participantes según los criterios de Brown (2015) para análisis independientes del AFE y el AFC. Al realizar este proceso, hemos garantizado la "no representatividad" de la muestra (Ferrando \& AnguianoCarrasco, 2010) en el momento de la confirmación factorial. También, se trató el AFE con matrices policóricas debido a la naturaleza de respuesta al ítem (Domínguez, 2014) y los ajuste del AFC corregidos para los casos en los que no se cumple la normalidad multivariante (Satorra \& Bentler, 2001; Hu \& Bentler, 1999; Byrne, 2006; Batista-Fogueta, Coenders, \& Alonso, 2004; Abad, Olea, Ponsoda, \& García, 2011).

Respecto a la fiabilidad de las pruebas se encontró resultados positivos y adecuados con respecto a la consistencia interna. Sin duda, la evaluación por medio del Alfa ordinal (Elosua \& Zumbo, 2008) arrojó valores favorables y pertinentes. La evidencia señala que el uso de $\alpha$ genera atenuación y disfraza el valor real de la fiabilidad como lo propuso Domínguez-Lara (2017). Se conluye que las pruebas CBQ y CBQ-V son consistentes para muestras ecuatorianas en términos de consistencia interna entre las puntuaciones de los ítems. 
A la luz de los hallazgos obtenidos se concluye que las pruebas de Cyberbullying (Calvete et al., 2010) y Cyberbullying-Victimización (Estévez et al., 2010) muestran estructuración factorial ajustada al contexto particular del Ecuador, además de fiable, por lo que su uso para análisis de cyberbullying en el Ecuador es pertinente. Sin embargo, requieren a futuro más estudios confirmatorios, en especial sobre la conformación de los distintos factores para cada prueba.

Las limitaciones del estudio se refieren a aspectos relacionados con el tipo de muestra con el que se trabajó; adolescentes con edades entre 14 a 19 años. Por lo que a futuro se recomienda realizar estudios a otros segmentos poblaciones como preadolescentes o estudiantes universitarios. También, el estudio se desarrolló en la ciudad de Ambato únicamente, por lo que se requerirán más estudios de otras zonas geográficas del país. Sobre las propiedades psicométricas, el presente trabajo no contempla más análisis sobre otros mecanismos de validación y fiabilidad debida a la extensión del trabajo presentado. Por lo que deben realizarse más estudios centrados en los elementos no considerados.

\section{Referencias}

Abad, F., Olea, J., Ponsoda, V., \& García, C. (2011). Medición en Ciencias Sociales y de la Salud. Madrid, España: Editorial SINTESIS S.A.

Ato, M., López, J., \& Benavente, A. (2013). Un sistema de clasificación de los diseños de investigación en psicología. Anales de Psicología, 29(3), 1038-1059. DOI: 10.6018/ analesps.29.3.178511.

Batista-Fogueta, M., Coenders, G., \& Alonso, J. (2004). Análisis factorial confirmatorio. Su utilidad en la validación de cuestionarios relacionados con la salud. Medicina Clínica, 122(Supl 1), 21-7.

Beckman, L., Hagquist, C., \& Hellström, L. (2012). Does the association with psychosomatic health problems differ between cyberbullying and traditional bullying? Emotional and Behavioural Difficulties, 17(3-4), 421-434. doi: 10.1080/13632752.20.

Brown, T. A. (2015). Confirmatory factor analysis for applied research (Vol. 2nd). New York, NY: Guilford Publications.

Buelga, S., \& Pons, J. (2012). Agresiones entre Adolescentes a través del Teléfono Móvil y de Internet. Psychosocial Intervention, 21(1), 91-101.

Buelga, S., Iranzo, B., Cava, M., \& Torralba, E. (2015). Psychological profile of adolescent cyberbullying aggressors. International Journal os Social Psychology, $30(2), 382-406$.

Byrne, B. M. (2006). Structural equation modeling with EQS. Basic concepts, applications, and programming (2nd ed.). Hills Dale, NJ: Lawrence Erlbaum Associates.

Calvete, E., Orue, I., Estévez, A., Villardón, L., \& Padilla, P. (2010). Cyberbullying in adolescents: Modalities and aggressors' profile. Computers in Human Behavior, 26(5), 1128-1135.

Actualidades en Psicología, 34(128), 2020, 35-50 
Castellanos, V., George, V., \& Gámez, M. (2017). Cyberbullying: A mental health problem among mexican adolescent. Vertientes Revista Especializada en Ciencias de la Salud, 19(1), 5-12.

Del Rey, R., Casas, J. A., Ortega-Ruiz, R., Schultze-Krumbholz, A., Scheithauer, H., Smith, P., . . G Guarini, A. (2015). Structural validation and cross-cultural robustness of the European Cyberbullying Intervention Project Questionnaire. Computers in Human Behavoir, 50, 141-147.

di Napoli, P. (2016). La violencia en las escuelas secundarias desde tres perspectivas de análisis. Hacia un estado del arte. Revista del Instituto de Estudios en Educación Universidad del Norte, 1(24), 61-84. doi: 10.14482/zp.24.8722.

Domínguez-Lara, S. (2014). Matrices policóricas/tetracóricas o matrices Pearson?: Un estudio metodológico. Revista Argentina de Ciencias del Comportamiento, 6(1), 39-48.

Dominguez-Lara, S. (2017). Fiabilidad y alfa ordinal. Actas Urológicas Españolas, 42(2), 140 141. doi: 10.1016/j.acuro.2017.07.002.

Eden, S., Heiman, T., \& Olenik-Shemesh, D. (2016). Bully versus victim on the internet: The correlation with emotional-social characteristics. Education and Information Technologies, 21(3), 699-713.

Elosua-Oliden, P., \& Zumbo, B. D. (2008). Coeficientes de fiabilidad para escalas de respuesta categorica ordenada. Psicothema, 20(4), 896-902.

Erdur-Baker, Ö. (2010). Cyberbullying and its correlation to traditional bullying, gender and frequent and risky usage of intenet-mediated communication tools. New Media Society, 12(1), 109-125. doi: 10.1177/1461444809341260.

Estévez, A., Villardón, L., Calvete, E., Padilla, P., \& Orue, I. (2010). Adolescentes víctimas de Cyberbullying: Prevalencia y características. Behavioral Psychology/Psicología Conductual, 18(1), 73-89.

Ferrando, P. J., \& Anguiano-Carrasco, C. (2010). El análisis factorial como técnica de investigación en psicología. Papeles del Psicólogo, 31(1), 18-33.

Fisher, B. W., Gardella, J. H., \& Teurbe-Tolon, A. R. (2016). Peer cybervictimization among adolescents and the associated internalizing and externalizing problems: a meta-analysis. Journal of youth and adolescence, 45(9), 1727-1743.

Flores Fernández, J. (2008). CiberBullying: Guia Rápida para la prevención del acoso por medio de las nuevas tecnologías. Bilbao, España: Ararteko. Recuperado de http://www. ararteko.net/RecursosWeb/DOCUMENTOS/1/1_1218_3.pdf

Gámez-Guadix, M., Villa-George, F., \& Calvete, E. (2014). Psychometric Properties of the Cyberbullying Questionnaire (CBQ) Among Mexican Adolescents. Violence and Victims, 29, 243-246.

Garaigordobil, M. (2011). Prevalencia y consecuencias del Cyberbullying: Una revisión. International Journal of Psychology and Psychological Theraphy, 11(2), 233-254. 
Garaigordobil, M. (2013). Cyberbullying. Screening de acoso entre iguales. Madrid, España: Tea Ediciones.

Garaigordobil, M. (2015). Ciberbullying en adolescentes y jóvenes del País Vasco: Cambios con la edad. Anales de Psicología, 31(3), 1069-1076.

Garaigordobil, M., \& Aliri, J. (2013). Ciberacoso en el País Vasco: Diferencias de sexo en víctimas, agresores y observadores. Behavioral Psychology / Psicología Conductual, 21(3), 461-474.

García, A., \& Jiménez, A. (2010). Estudio exploratorio y descriptivo del Cyberbullying en escolares de secundaria. Revista Qurriculum, 23, 155-164.

García, L., \& Orellana, O. (2010). Cyberbullying en escolares de educación secundaria de Lima Metropolitana. Revista de Investigación en Psicología, 13(2), 83-99.

Hu, L., \& Bentler, P. M. (1999). Cutoff criteria for fit indexes in covariance structure analysis: Conventional criteria versus new alternatives. Structural Equation Modeling, 6(1), 1-55.

Instituto Nacional de Estadísticas y Censos [INEC]. (2015). Fasciculo Provincial Tungurahua. Quito: INEC.

Larzabal-Fernández, A., Ramos-Noboa, M. I., \& Hong, A. E. (2019). El cyberbullying y su relación con el estrés percibido en estudiantes de bachillerato de la provincia de Tungurahua. Ciencias Psicológicas, 13(1), 150-157. doi: 10.22235/ cp.v13i1.1815.

Lorenzo-Seva, U., \& Ferrando, P. (2013). FACTOR 9.2 A Comprehensive Program for Fitting Exploratory and Semiconfirmatory Factor Analysis and IRT Models. Applied Psychological Measurement, 37(6), 497-498.

McDonald, R. P. (1999 ). Test theory: A unified treatment. Mahwah, New Jersey: Lawrence Erlbaum Associates, Inc.

Moreta, R., Reyes, C., Mayorga, M., \& León-Tamayo, L. (2017). Estimación sobre niveles y factores de riesgo psicosocial en adolescentes escolarizados de Ambato, Ecuador. Pensando Psicología, 13(22), 29-40.

Moreta-Herrera, C. R., Poveda-Ríos, S., \& Ramos-Noboa, I. (2018). Indicadores de violencia relacionados con el ciberbullying en adolescentes del Ecuador. Pensando Psicología, 14(24), 1-12. doi: 10.16925/pe.v14i24.1895.

Olweus, D. (1998). Conductas de acoso y amenaza entre escolares. Madrid, España: Morata.

Ortega, R., Calamaestra, J., \& Mora, J. (2008). Cyberbullying. International Journal of Psychology and Psychological Therapy, 8(2), 183-192.

Ortega-Reyes, J., \& González-Bañales, D. (2015). Impacto del Cyberbullying en el rendimiento académico de estudiantes de nivel medio superior. Mexico: Instituto Universitario Anglo Español.

Peña, A. Q., Urday, W. M., \& Soto, C. M. (2013). Estilos de crianza y empatía en adolescentes implicados en ciberbullying. Revista de Investigación en Psicología, 12(6), 61-87. 
R Core Team. (2015). R: A language and environment for statistical computing. Vienna, Austria: $\mathrm{R}$ Foundation for Statistical Computing.

Resett, S. A. (2016). Cyberbullying, correlatos psicológicos y diferencias de sexo en una muestra de adolescentes Argentinos. Investigaciones en Psicología, 21(3), 1-31.

Resett, S., \& Gámez-Guadix, M. (2018). Propiedades psicométricas del cuestionario de ciberbullying en una muestra de adolescentes argentinos. Universitas Psychologica, 17(5), 1-12. doi: 10.11144/Javeriana.upsy17-5.ppcc.

Satorra, A., \& Bentler, P. M. (2001). A scaled difference chi-square test statistic for moment structure analysis. Psychometrika, 66(4), 507-514.

Tokunaga, R. S. (2010). Following you home from school: A critical review and synthesis of research on cyberbullying victimization. Computers in Human Behavior, 26, 277-287.

Varela, J., Perez, C., Schwaderer, H., Astudillo, J., \& Lecannelier, F. (2014). Caracterización de Cyberbullying en el gran Santiago de Chile, en el año 2010. Psicología Escolar e Educacional, 18(2).

Ventura, J., \& Caycho, T. (2016). Análisis psicométrico de una escala de dependencia emocional en universitarios peruanos. Revista de Psicología, 25(1), 1-17. doi: 10.5354/0719-0581.2016.42453.

Wachs, S. (2012). Moral disengagement and emotional and social difficulties in bullying and cyberbullying: Differences by participant role. Emotional and Behavioural Difficulties, 17(3-4), 347-360. doi: 10.1080/13632752.2012.704318.

You, S., Lee, Y., \& Kim, E. (2016). Physical, social, and cyberbullying: Relationships with adolescents' psychosocial factors. Child Indicators Research, 9(3), 805-823. 


\section{Anexo 1}

Cyberbullying Questionnaire (CBQ)

Perpetración

1. He enviado mensajes amenazantes o insultantes por medio de correo electrónico

2. He enviado mensajes amenazantes o insultantes

3. He excluido a alguien de un grupo en línea

4. He subido imágenes humillantes de un compañero de clase en internet

5. He enviado enlaces electrónicos de imágenes humillantes a otras personas

6. He obtenido la contraseña y enviado correos electrónicos hacia otros en nombre de esa persona, lo cual puede hacer que esa persona quede mal o cause problemas

7. He grabado un video o tomado fotos por celular mientras un grupo se ríe y obliga a la otra persona a hacer algo humillante o ridículo

8. He enviado dichos videos a otra persona

9. He grabado un video o tomado fotos por celular mientras alguien golpea o lastima a otra persona

10. He enviado dichas imágenes grabadas a otras personas

11. He enviado mensajes que incluyan amenazas o que son muy intimidantes repetitivamente

12. He grabado un video o tomado fotos por celular de algún compañero de clase mientras él o ella está llevando a cabo un comportamiento de connotación sexual

13. He enviado dichas imágenes a otras personas

14 He transmitido en línea los secretos de otras personas, información comprometida o imágenes

\section{Cuestionario de victimas de Cyberbullying $(C B Q-V)$}

Victimización

1. Han difundido en internet información confidencial mía

2. Me han grabado realizando conductas sexuales

3. Me han grabado un video o me han fotografiado mientras me obligaban a hacer algo humillante

4. Me han grabado un video o me han fotografiado mientras me agredían físicamente

5. He recibido mensajes amenazantes o insultantes

6. Han colgado imágenes mías humillantes en internet

7. Han colgado comentarios sobre mí en internet

8. Han usado mi identidad

9. He recibido masivamente mensajes muy amenazantes 